\title{
A DIFFUSED INTERFACE IMMERSED BOUNDARY-LATTICE BOLTZMANN METHOD FOR SIMULATION OF STENOSIS
}

\author{
QIUXIANG HUANG, LI WANG, FANGBAO TIAN, JOHN YOUNG AND JOSEPH C. \\ S. LAI
}

School of Engineering and Information Technology, University of New South Wales Canberra, ACT 2600, Australia.

Emails: qiuxiang.huang@student.adfa.edu.au (QH), l.wang@unsw.edu.au (LW), f.tian@adfa.edu.au (FBT), j.young@adfa.edu.au (JY) and j.lai@ adfa.edu.au (JCSL).

Key words: Immersed boundary method, Lattice Boltzmann method, Stenosis, Hemodynamics

\begin{abstract}
The immersed boundary method has attracted growing interest in CFD research community due to its simplicity in dealing with moving boundaries. In the diffused interface immersed boundary method, a discrete delta function is introduced to account for the boundary effects on the fluid, which causes the diffusion of the boundary interface. Therefore, the diffused interface immersed boundary method requires higher grid resolution in the vicinity of the immersed boundaries to get a better representation of the boundary. A strategy for the application of diffused interface immersed boundary-lattice Boltzmann method is introduced in the simulation of stenosis. The developed numerical method has been examined in the simulation of stenosis. Results show that the current solver is able to accurately predict the velocity profile within the stenosis.
\end{abstract}

\section{INTRODUCTION}

Computational fluid dynamics become an important tool for improving our understanding the effect of normal physiological and pathological behaviors in the arterial system [1]. Stenosis, in ideal circumstances, is modeled as a straight rigid pipe with a constriction. A range of studies have been conducted based on this model, including experimental study by Ahmed \& Giddens [2], two-dimensional (2D) numerical studies by Tian et al. [3] and Huang et al. [4] and three-dimensional (3D) numerical studies by Pal et al. [5] and Varghese et al. [6].

In the diffused interface immersed boundary method (IBM), a discrete delta function is introduced to account for the boundary effects on the fluid, which causes the diffusion of the boundary interface. Thus, the diffused interface IBM is first order scheme [7] and requires higher grid resolution in the vicinity of the immersed boundary to get a better representation of the boundary. Liu et al. [8] used the diffused interface IBM for the simulation of flapping foil. Wang et al. $[9,10]$ adopted the diffused interface IBM for the simulation of heat transfer and fluid-structure interaction of a flapping filament. Pal et al. [5] used a second order IBM in the simulation of turbulent flow in an idealized stenotic blood vessel. However, there are few studies on the diffused interface IBM in the simulation of blood flow. In this work, a strategy for the application of diffused interface immersed boundary-lattice Boltzmann method (IB-LBM) is introduced here for the simulation of stenosis. 


\section{GOVERNING EQUATIONS}

The unsteady incompressible flow is governed by the continuity and Navier-Stokes equations:

$$
\nabla \cdot \boldsymbol{u}=0, \quad \frac{\partial \boldsymbol{u}}{\partial t}+\boldsymbol{u} \cdot \nabla \boldsymbol{u}=-\frac{1}{\rho} \nabla p+v \nabla^{2} \boldsymbol{u}+\boldsymbol{f},
$$

where $\boldsymbol{u}$ is the fluid velocity, $\rho$ is the constant density, and $p$ and $v$ are respectively the pressure and kinematic viscosity, and $f$ is the body force.

\section{NUMERICAL METHOD}

The D3Q19 lattice Boltzmann method (LBM) with multi-relaxation-time (MRT) model is adopted for the fluid dynamics. The IBM is used to realize the no slip and no penetration boundary conditions of the rigid tube wall. In the LBM, the macroscopic dynamics of the fluid are the result of the statistical behaviour of the particles, which are described by the distribution function $g_{i}(\boldsymbol{x}, t)$. The evolution of this distribution function is according to

$$
g_{i}\left(x+e_{i} \Delta t, t+\Delta t\right)-g_{i}(x, t)=\Omega_{i}(x, t)+\Delta t G_{i},
$$

where $g_{i}(\boldsymbol{x}, t)$ is the distribution function for particles with velocity $\boldsymbol{e}_{i}$ at position $\boldsymbol{x}$ and time $t, \Delta t$ is the time increment, $\Omega_{i}(\boldsymbol{x}, t)$ is the collision operator, and $G_{i}$ is the forcing term accounting for the body force $f$. The D3Q19 model [11] is used on a square lattice. The MRT collision model is adopted and is given by [12]:
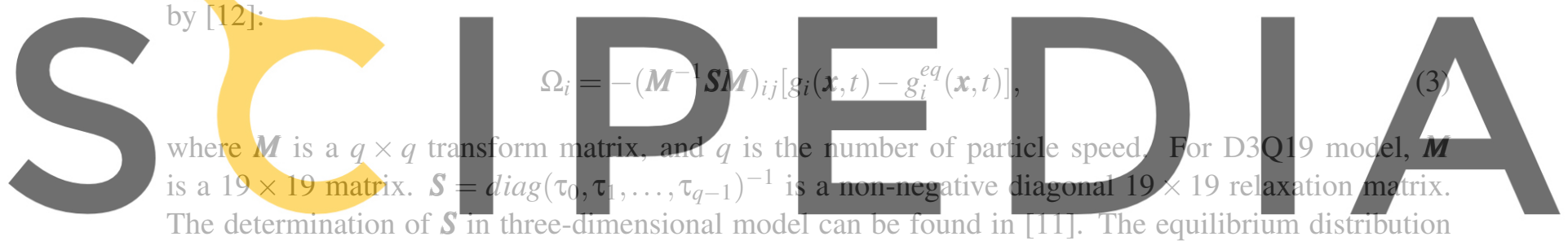

The determination of $S$ in

$$
g_{i}^{e q}=\rho \omega_{i}\left[1+\frac{e_{i} \cdot u}{c_{s}^{2}}+\frac{u u:\left(e_{i} e_{i}-c_{s}^{2} \boldsymbol{I}\right)}{2 c_{s}^{4}}\right]
$$

where $c_{s}=\Delta x /(\sqrt{3} \Delta t)$ is the speed of sound, $\Delta x$ is the lattice spacing, $\boldsymbol{I}$ is the unit tensor, and the weighting factors $\omega_{i}$ are given by $\omega_{0}=1 / 3, \omega_{1-6}=1 / 18$ and $\omega_{7-18}=1 / 36$. The velocity $\boldsymbol{u}$, mass density $\rho$ and pressure $p$ can be obtained according to

$$
\rho=\sum_{i} g_{i}, \quad p=\rho c_{s}^{2}, \quad \boldsymbol{u}=\left(\sum_{i} \mathbf{e}_{i} g_{i}+\frac{1}{2} f \Delta t\right) / \rho,
$$

The force scheme proposed in [14] is adopted to determine $G_{i}$

$$
\begin{array}{r}
G_{i}=\left[\boldsymbol{M}^{-1}(\boldsymbol{I}-\boldsymbol{S} / 2) \boldsymbol{M}\right]_{i j} F_{i}, \\
F_{i}=\left(1-\frac{1}{2 \tau}\right) \omega_{i}\left[\frac{\boldsymbol{e}_{i}-\boldsymbol{u}}{c_{s}^{2}}+\frac{\boldsymbol{e} \cdot \boldsymbol{u}}{c_{s}^{4}} \boldsymbol{e}_{i}\right] \cdot \boldsymbol{f},
\end{array}
$$

where $\tau$ is the non-dimensional relaxation time. 
In the present study, an diffused interface IBM is adopted to handle the no slip and no penetration of the rigid tube walls. A comprehensive overview of this method was given by Kang et al. [15]. In this method, a body force $f$ is added in the Navier-Stokes equation to mimic a boundary condition according to,

$$
\begin{gathered}
\boldsymbol{f}(\boldsymbol{x}, t)=-\int \boldsymbol{F}_{i b}(s, t) \boldsymbol{\delta}(\boldsymbol{x}-\boldsymbol{X}(s, t)) d A, \\
\boldsymbol{F}_{i b}(s, t)=2\left(\boldsymbol{U}_{i b}(s, t)-\boldsymbol{U}(s, t)\right), \\
\boldsymbol{U}_{i b}(s, t)=\int \boldsymbol{u}(x, t) \boldsymbol{\delta}(\boldsymbol{X}(s, t)-\boldsymbol{x}) d \boldsymbol{x}
\end{gathered}
$$

where $\boldsymbol{F}_{i b}(s, t)$ is the Lagrangian force density, $d A$ is the element surface area of the immersed boundary, $\boldsymbol{\delta}(\boldsymbol{x}-\boldsymbol{X}(s, t))$ is Dirac's delta function, $\boldsymbol{x}$ is the coordinate of the fluid lattice nodes, $\boldsymbol{X}$ is the coordinate of the tube wall, $\boldsymbol{U}_{i b}(s, t)$ is the immersed boundary velocity obtained by interpolation at the immersed boundary, and $\boldsymbol{U}(s, t)=0$ for a rigid tube wall. The 4-point discrete delta function $\boldsymbol{\delta}_{h}(\boldsymbol{x})$ is used to approximate the Dirac delta function [13]:

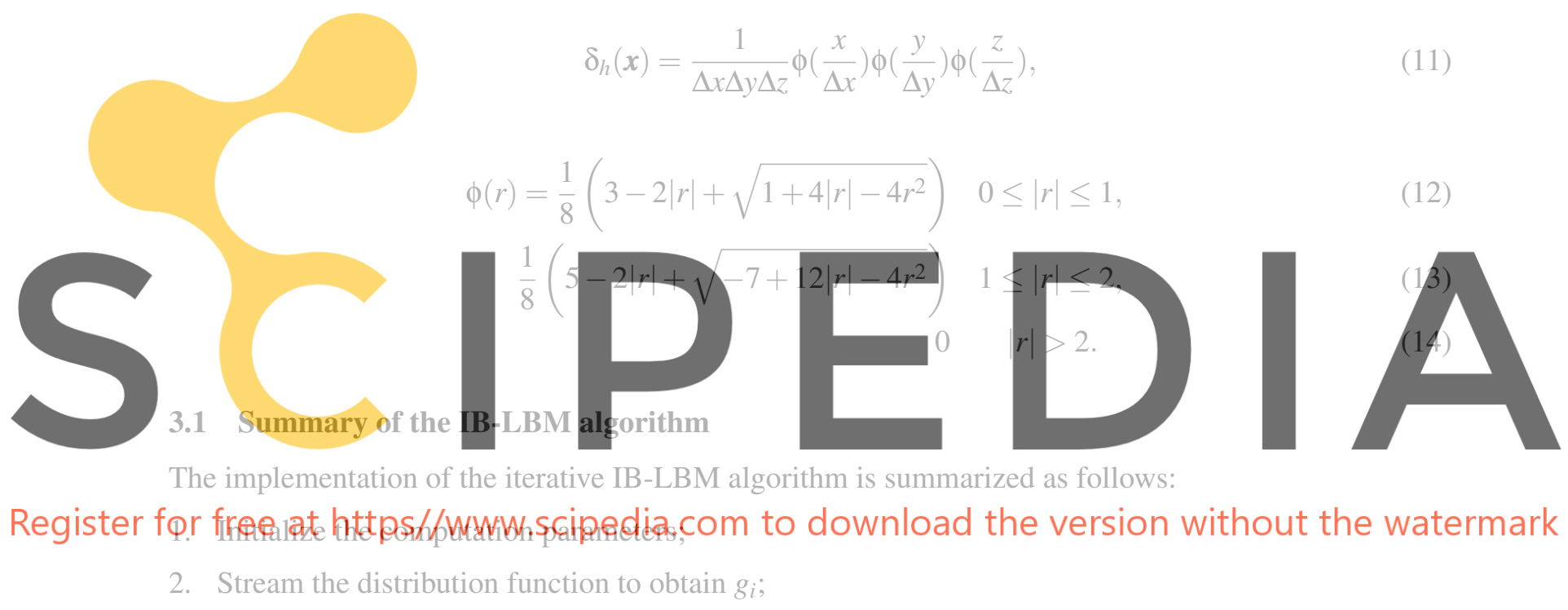

3. Compute the macroscopic variables: density $\rho$ and the uncorrected velocity $\boldsymbol{u}$ using

$$
\rho=\sum_{i} g_{i}, \quad \boldsymbol{u}=\frac{1}{\rho} \sum_{i} \boldsymbol{e}_{i} g_{i}
$$

4. Set iteration counter $m$ to 0 ;

5. Interpolate the immersed boundary velocity $\boldsymbol{U}_{i b}^{m}$ using equation (10);

6. Compute the Lagrangian force density $\boldsymbol{F}_{i b}^{m}(s, t)$ using equation (9);

7. Spread $\boldsymbol{F}_{i b}^{m}(s, t)$ to the Eulerian lattice to obtain $\boldsymbol{f}^{m}(\boldsymbol{x})$;

8. Correct Eulerian velocity near to the immersed boundary according to

$$
\boldsymbol{u}^{m+1}(\boldsymbol{x})=\boldsymbol{u}^{m}(\boldsymbol{x})+\frac{\boldsymbol{f}^{m}(\boldsymbol{x}) d t}{2 \rho(\boldsymbol{x})}
$$




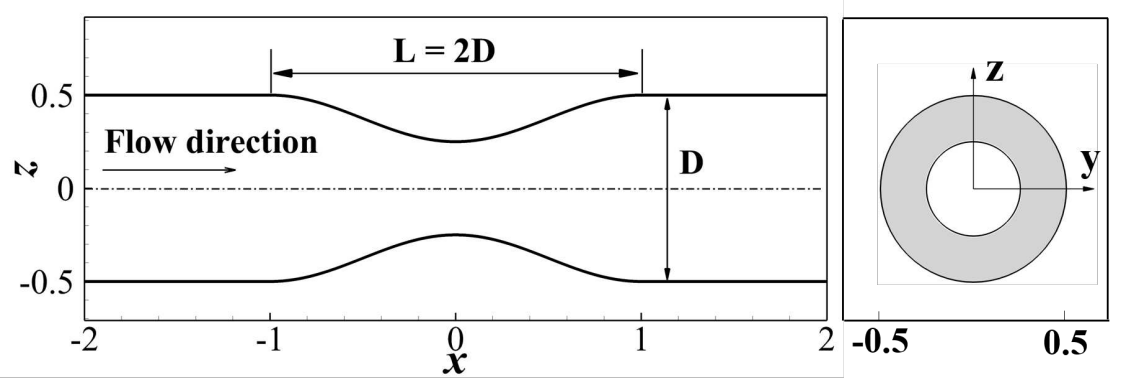

Figure 1: Side and front views of the stenosis geometry.

9. Update iteration counter $m=m+1$;

10. Repeat steps 5-9 until the immersed boundary velocity $\boldsymbol{U}_{i b}^{m}$ converges to the velocity of the tube wall $U(s, t)$;

11. Calculate $g_{i}^{e q}$ using equation (4);

12. Perform the collision step with the total Eulerian body force:

$$
f(x)=\sum_{m=1}^{m_{\max }} f^{m}(x)
$$
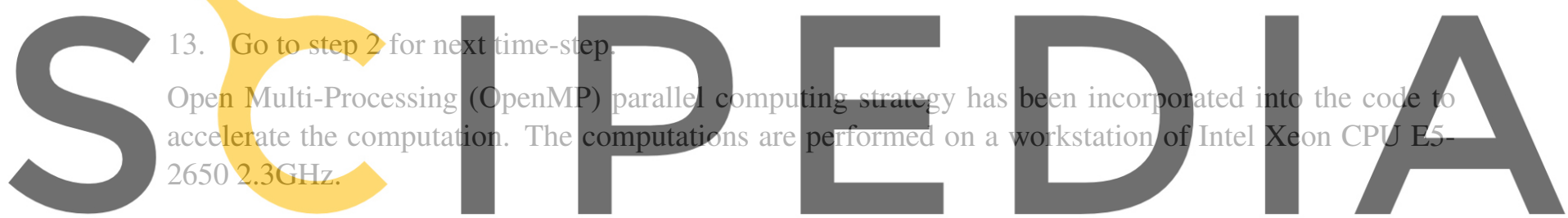

4 RESULTS

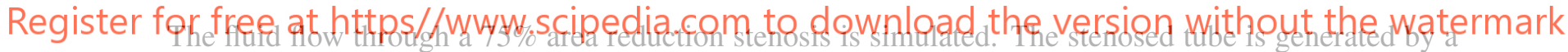
cosine function of the axial coordinate $\mathrm{x}$ :

$$
\left.\begin{array}{l}
\begin{array}{l}
S(x)=\frac{1}{2} D\left[1-s_{o}\left(1+\cos \left(2 \pi\left(x-x_{o}\right) / L\right)\right)\right] \\
y=S(x) \cos \theta, \quad z=S(x) \sin \theta
\end{array}
\end{array}\right\}
$$

where $D$ is the diameter of the non-constricted tube, $L=2 D$ is the length of the stenosis, for a $75 \%$ area reduction stenosis $s_{o}=0.25$ and $x_{o}$ is the central location of the stenosis. Figure 1 shows the side and front view of the stenosis. A steady Hagen-Poiseuille velocity profile is imposed at the inlet, and the initial flow field is initialized as:

$$
\frac{u}{U_{0}}=2\left(1-r^{2}\right), \quad \frac{v}{U_{0}}=0, \quad \frac{w}{U_{0}}=0,
$$

where $u, v$ and $w$ are the velocity in $x-, y-$ and $z$-directions, respectively, $U_{0}$ is the averaged velocity at the inlet and $r=\sqrt{y^{2}+z^{2}}$ is the radial distance from the tube centreline. The computational domain is a rectangular parallelopiped $(x \in[-3 D, 17 D], y \in[-0.6 D, 0.6 D]$ and $z \in[-0.6 D, 0.6 D])$. The grid size of the fluid and the tube are $0.02 D$ and $0.01 D$, respectively. The Reynolds number $R e=500$ is defined 


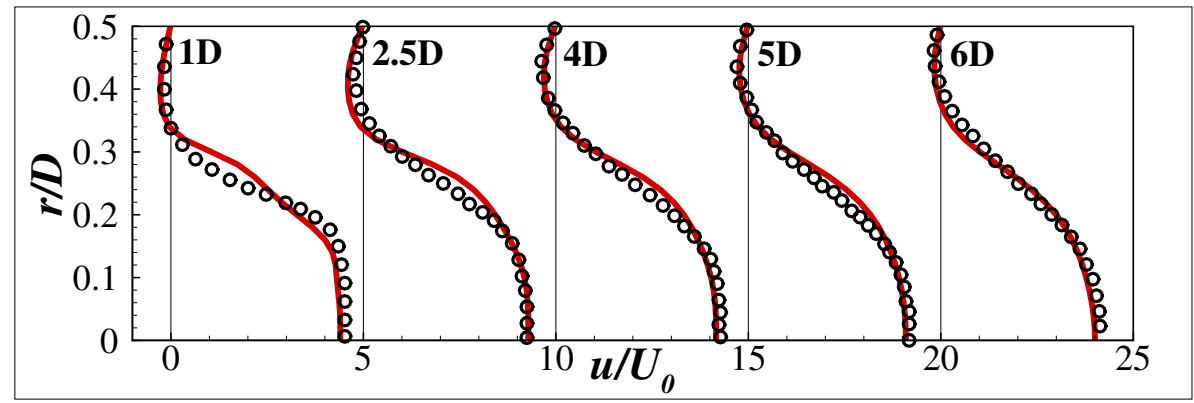

Figure 2: Axial velocity profiles at five axial locations $(x=1 D, x=2.5 D, x=4 D, x=5 D$, and $x=6 D)$ downstream of the stenosis. Red solid: present IB-LBM; black circle: direct numerical simulation by Varghese et al. [6]. The tube wall is extended outwards by one grid point to compensate the diffusion of the boundaries caused by the diffused interface IBM.

based on the diameter $D$ and the averaged velocity $U_{0}$ at the inlet. The tube wall is rigid with the no-slip boundary condition and a constant pressure is specified at the outlet. The tube wall is extended outwards by one grid point to compensate the diffusion of the boundaries caused by the diffused interface IBM.

As shown in figure 2, the axial velocity profiles at five axial locations downstream of the stenosis shows good agreement with the direct numerical simulation results by Varghese et al. [6]. The predicted pressure, streamwise velocity and vorticity contours are shown in figure 3. The flow is laminar and symmetric with a jet formed downstream of the stenosis. Figure 3(a) shows that the velocity increase significantly due to the cor the most constricted part that the flow is symmetific

5 CONCLUSIONS
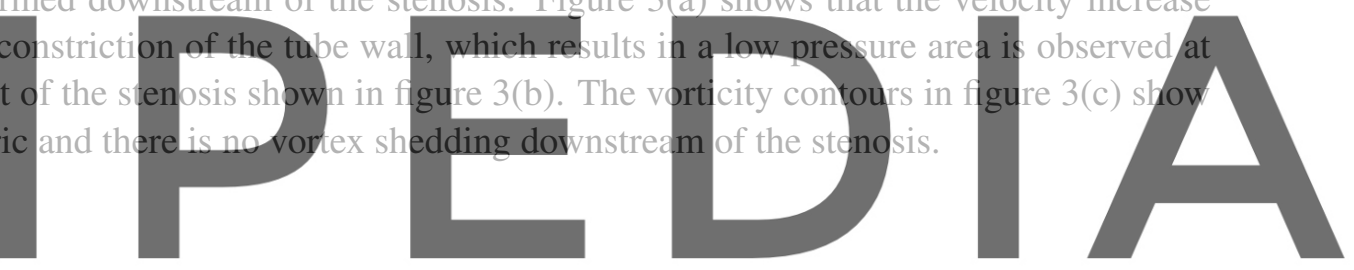

A strategy for the application of diffused interface IB-LBM is firstly introduced in the simulation of

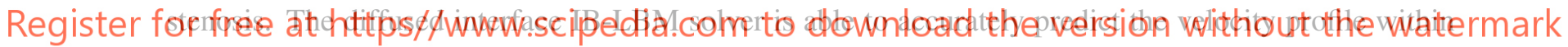
the stenosis.

\section{ACKNOWLEDGEMENTS}

Mr. Q. Huang acknowledges the support of the University International Postgraduate Award by the University of New South Wales. Dr. F.-B. Tian is the recipient of an Australian Research Council Discovery Early Career Researcher Award (project number DE160101098). The computation work of this research was partially performed on the National Computational Infrastructure (NCI) supported by the Australian Government.

\section{REFERENCES}

[1] Huang, Q., Sun, J., and Xu, C. (2020). Effects of waveform shape of pulsatile blood flow on hemodynamics in an artery bifurcation model. Proceedings of the Institution of Mechanical Engineers, Part C: Journal of Mechanical Engineering Science, 0954406220911397.

[2] Ahmed, S. A., Giddens, D. P. (1983). Velocity measurements in steady flow through axisymmetric 

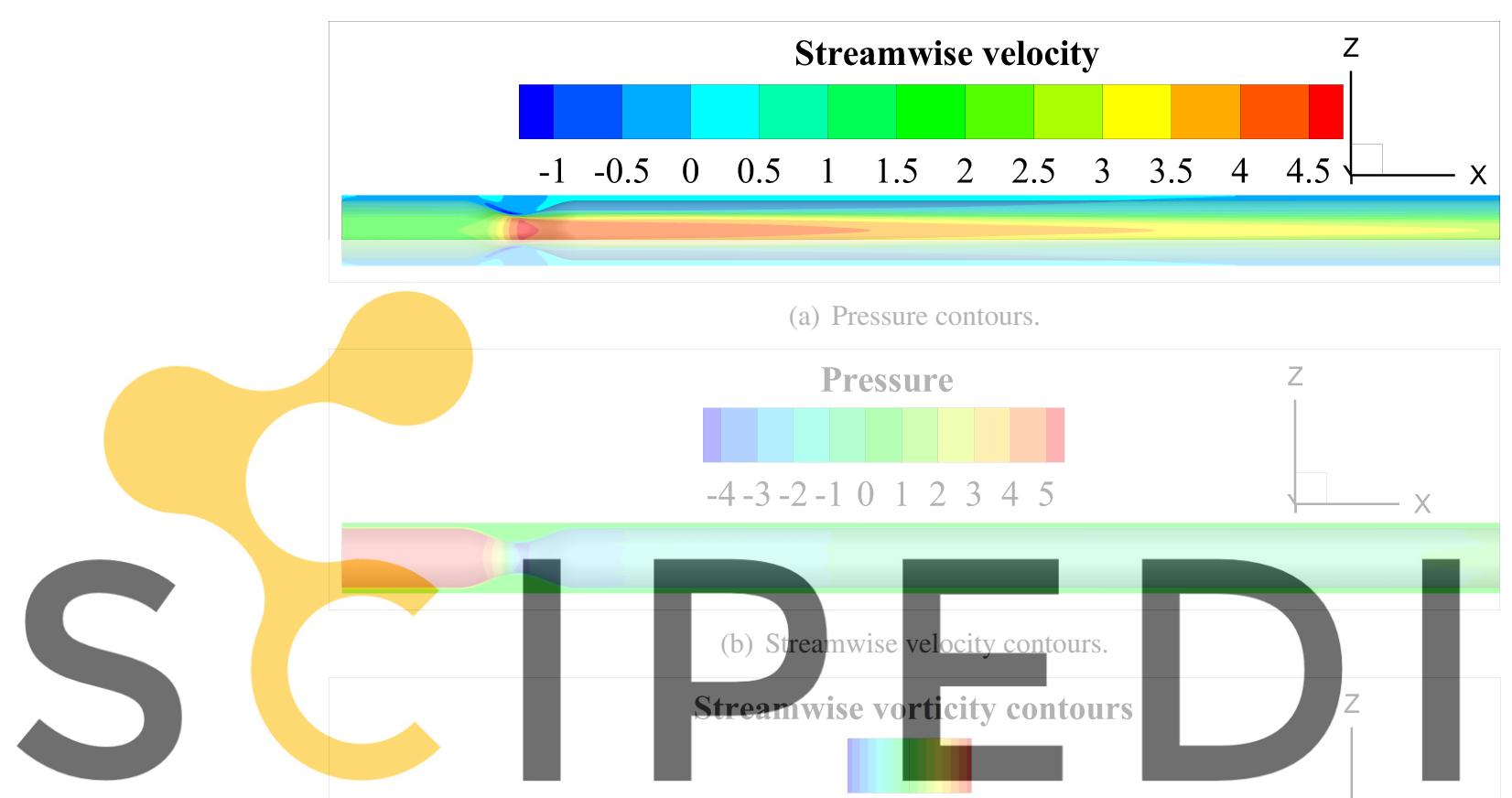

(a) Pressure contours.

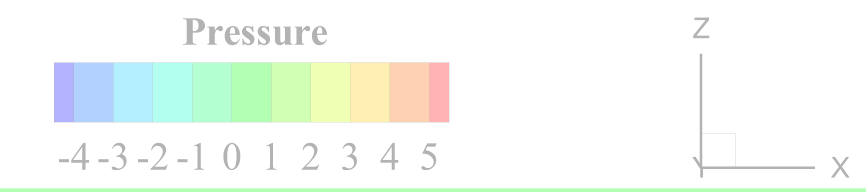

\section{Register for free at https//www.scipedia.com to download the version without the watermark}

(c) Streamwise vorticity contours.

Figure 3: Pressure, streamwise velocity and vorticity contours in the Z-X symmetry plane of the $75 \%$ area reduction stenosis. The velocity is non-dimensionalized by the inlet averaged velocity $U_{0}$. The pressure here is a relative pressure to the outlet pressure and is non-dimensionalized by $\rho U_{0}^{2}$. The vorticity is non-dimensionalized by $U_{0} / D$. 
stenoses at moderate Reynolds numbers. Journal of Biomechanics, 16(7): 505-516.

[3] Tian, F.-B., Zhu, L., Fok, P. W., et al. (2013). Simulation of a pulsatile non-Newtonian flow past a stenosed 2D artery with atherosclerosis. Computers in Biology and Medicine, 43(9): 1098-1113.

[4] Huang, Q., Tian, F.-B., Young, J. and Lai, J. C. S. A diffused interface immersed boundary-lattice Boltzmann method for simulation of channel flow. 22nd Australasian Fluid Mechanics Conference AFMC2020, Brisbane, Australia, 7-10 December 2020. Brisbane, Australia

[5] Pal, A., Anupindi, K., Delorme, Y., et al. (2014). Large eddy simulation of transitional flow in an idealized stenotic blood vessel: evaluation of subgrid scale models. Journal of Biomechanical Engineering, 136(7).

[6] Varghese, S. S., Frankel, S. H., Fischer, P. F. (2014).Direct numerical simulation of stenotic flows. Part 1. Steady flow. Journal of Fluid Mechanics, 582(1): 253-280.

[7] Huang, W.-X, Tian, F.-B. (2019). Recent trends and progresses in the immersed boundary method. Proceedings of the Institution of Mechanical Engineers, Part C: Journal of Mechanical Engineering Science, 233: 7617-7636.

[8] Liu, Z., Tian, F.-B., Young, J., and Lai, J. C. (2017). Flapping foil power generator performance enhanced with a spring-connected tail. Physics of Fluids, 29(12): 123601.

[9] Wang, L., Tian, F.-B. (2018). Heat transfer in non-Newtonian flows by a hybrid immersed boundary-lattice Boltzmann and finite difference method. Applied Sciences, 8(4): 559.

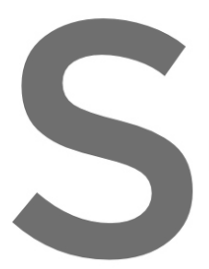

[10] Wang, L., Tian, F.-B vicinity of a rigid wat

11] d'Humieres, D. (2002 Philosophical Trans Engineering Science
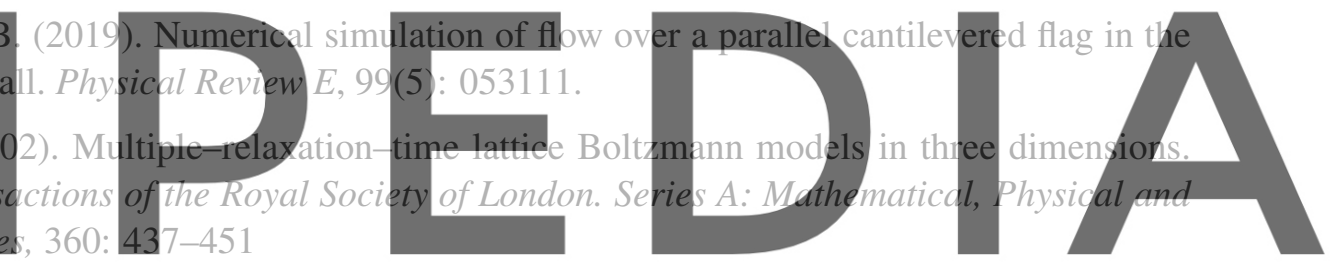

[12] Lallemand, P., Luo, L. S. (2000). Theory of the lattice Boltzmann method: Dispersion, dissipation,

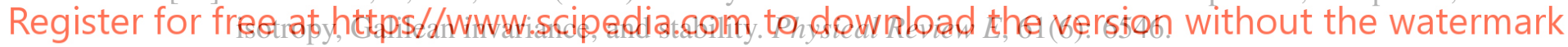

[13] Peskin, C. S.. (2002). The immersed boundary method. Acta numerica, 11: 479-517.

[14] Guo, Z. L., Zheng, C. G., Shi, B. C. (2002). Non-equilibrium extrapolation method for velocity and pressure boundary conditions in the lattice Boltzmann method. Chinese Physics, 11(4): 366.

[15] Kang, S. K., Hassan, Y. A. (2011). A comparative study of direct-forcing immersed boundary lattice Boltzmann methods for stationary complex boundaries. International Journal for Numerical Methods in Fluids, 66(9): 1132-1158. 